Article

\title{
Controlled Release on Cardiovascular Stents Using Plasma-Enhanced Adhesion of Biodegradable Nanoparticles
}

\author{
Alberto Ponce, Víctor Ramos-Pérez, Salvador Borrós * \\ Grup d'Enginyeria de Materials (GEMAT), Institut Químic de Sarrià, Universitat \\ Ramon Llull, Barcelona 08017, Spain \\ * Correspondence: Salvador Borrós, Email: salvador.borros@iqs.url.edu.
}

\begin{abstract}
One of the most common solutions to treat ischemic heart disease nowadays is the implantation of drug eluting stents. Currently, new strategies are being developed to improve healing process, which includes pharmacological treatments or gene therapy. In this paper, we presented a proposal based on the use of poly( $\beta$-amino ester) (pBAE) nanoparticles. To enhance the release method, we used an approach based on attaching these nanoparticles on a polymeric coating. The process includes coating metallic cardiovascular stents with a thin and highly functionalized layer of pentafluorophenyl methacrylate (PFM) to which loaded nanoparticles are chemically bonded. Through this design, when a stent is expanded during its implantation, nanoparticles will stay attached to the polymer matrix. Nanocarriers will penetrate target adjacent cells, guaranteeing effective drug delivery. Results obtained show that this work opens a pathway for pharmacological and/or gene delivery systems based on the adhesion of pBAE nanoparticles prior to stent implantation.
\end{abstract}

KEYWORDS: control release; cardiovascular stent; pentafluorophenyl methacrylate (PFM); gene delivery; poly( $\beta$-amino ester) (pBAE)

$\begin{array}{ll}\text { ABBREVIATIONS } \\ \text { CAD } & \text { Coronary Artery Disease } \\ \text { CDCP } & \text { Centres for Disease Control and Prevention } \\ \text { DES } & \text { Drug eluting stents } \\ \text { BMS } & \text { Bare metal stent } \\ \text { PBAE } & \text { Poly( } \beta \text {-amino ester }) \\ \text { MiRNA } & \text { Micro RNA } \\ \text { SiRNA } & \text { Small interfering RNA } \\ \text { RNAi } & \text { Interfering RNA } \\ \text { PFM } & \text { Pentafluorophenyl methacrylate } \\ \text { GFP } & \text { Green fluorescent plasmid } \\ \text { PGFP } & \text { Green fluorescent protein vector } \\ \text { SEM } & \text { Scanning electron microscopy } \\ \text { AFM } & \text { Atomic force microscopy }\end{array}$




$\begin{array}{ll}\text { BSA } & \text { Bovine serum albumin } \\ \text { IR } & \text { Infrared } \\ \text { ATR } & \text { Attenuated Total Reflection } \\ \text { RF } & \text { Radiofrequency } \\ \text { DC } & \text { Duty cycles } \\ \text { DMEM } & \text { Dulbecco's modified Eagle medium } \\ \text { FBS } & \text { Fetal bovine serum } \\ \text { Ra } & \text { Roughness average } \\ \text { Rq } & \text { Root mean square roughness } \\ \text { Rmax } & \text { Maximum roughness } \\ \text { Rz } & \text { Mean roughness depth }\end{array}$

\section{INTRODUCTION}

Coronary Artery Disease (CAD), also known as ischemic heart disease, is the most typical type of heart disease, killing more than 385,000 people annually according to Centres for Disease Control and Prevention (CDCP) [1]. It is a serious and common disease that can profoundly influence a patient's prognosis and quality of life due to the consequent restriction of blood supply (ischemia). The mechanism of coronary ischemia is described as the development of coronary atherosclerosis, while the atherosclerotic process is not well understood [2,3]. Atherosclerosis is associated with inflammatory processes in the endothelial cells of the vessel wall, and related with the deposition of fatty substances (cholesterol, fatty acids, etc.) in arteries, resulting in a narrowed blood vessel lumen (stenosis) and thus reduced myocardial oxygen supply [2,4]. To solve this issue, a common approach is to undergo a coronary stent implantation. This procedure is used to achieve a permanent dilation of a stenotic coronary artery in order to improve myocardial perfusion. During the process of reopening the artery lumen and restoring the exterior layer of the artery wall, there are various treatments used to ensure positive results. Although there are different approaches, these are far from being optimal. Besides the risk of subacute thrombosis, the main problem of stents is late intra-stent restenosis which can be caused by multiple factors. The stent acts as a platform for preventing acute vascular occlusion, removing the elastic recoil and reducing late remodelling. Despite the structural benefit brought by stents, neointimal proliferation and hyperplasia persist and can even be stimulated by the stent itself [5-10].

To mitigate these effects, during a procedure of stent implantation, a simultaneous pharmacological treatment is often performed. In this case, there are different types of stents which can be used to treat the affected area $[11,12]$.

The most common solutions, with overall good results, imply drug eluting stents (DES) [5-10]. The first DES developed were the Taxus and Cypher stents, incorporating paclitaxel and sirolimus, respectively. Both stents demonstrated a marked inflammatory reduction compared to bare metal stents (BMS), without complete inhibition of restenosis (TAXUS I, II, 
II and IV, SIRIUS, E-SIRIUS and C-SIRIUS studies) [11-15]. Drugs used in DES might include inhibitors of inflammation, platelet aggregation, cell migration and proliferation, or promoters of vascular healing and reendothelization. Although there are a variety of DES present in the market, none presents an optimal model which solves all the derived consequences of stent application.

Due to the lack of a unique solution, a variety of different methods based on drug delivery systems and gene therapy systems have been tested out. Results from these drug eluting stents have been obtained by elution analysis of pharmacological release [16-18]. A second approach, gene therapy, is a technique that uses nucleic acids to treat or prevent diseases by acting on the genetic roots of the illness [19,20]. The goal of gene therapy is to modify a gene, or genetic pathway, to prevent or treat a disease. Although gene therapy can be applied with DES treatment, it would render better results if eluted with a carrier that ensured a controlled delivery. There have been different techniques developed to treat inflammatory responses through gene modification, but these efforts have not been directed to achieve restenosis reduction through controlled release with DES. One of the most promising carriers used which enables safe and efficient delivery systems are nanoparticles [13,21-24].

When applying nanoparticles locally, they can penetrate the vessel wall and form a depot which will allow a sustained release of drugs into the arterial wall. This method allows a focused treatment which targets affected cells [25]. Nanocarriers can be tailored to have specific sizes, shapes, densities or functionalities. Customization makes nanoparticles ideal as drug delivery vehicles when used to encapsulate desired drugs or gene vectors. It is of vital importance to ensure the non-toxicity of the nanoparticles used for drug delivery, as they are going to be used to penetrate the targeted cells [26]. As mentioned before, nanoparticles can be excellent carriers and can prove useful when developing a therapeutic strategy for DES. Achieving a controlled release of encapsulated contents is possible as long as adhesion of nanoparticles to a stent surface is guaranteed. A good range of biodegradable and biocompatible materials have been tried out to fulfil this task, showing there is no unique solution to this question. Up to date, DES have been made of different polymers, but some of the most used ones are poly(lactic acid) and poly(lactic-co-glycolic acid) [27-32].

In this study, a promising approach has been implemented to fulfil therapeutic delivery of DES through the adhesion of poly( $\beta$-amino ester) (pBAE) nanoparticles to the stent strut. If this system brings positive results, on one hand, it will be useful to deliver antiproliferative drugs. On the other hand, it will also be useful to ensure controlled and localized release of a large number of microRNA (miRNA). These endogenous, noncoding, single-stranded RNAs have been demonstrated to reduce proliferation of smooth muscle cells, which is a key factor for stent treatment. miRNAs are novel regulatory RNAs for neointimal lesion 
formation, making them a useful tool for the treatment of proliferative vascular diseases such as atherosclerosis, postangioplasty restenosis, transplantation arteriopathy, and stroke [33]. Our approach can also enhance the therapeutic treatment, as it provides a regulated release of small interfering RNA (siRNA), which have an important role on regulating the inflammatory process [34]. This tailored release is able to span over an initial burst, producing a better controlled pharmacological liberation.

pBAEs used in this study have previously shown high transfection efficiencies, biodegradability and biocompatibility. Furthermore, nanoparticles made from these polymers can be tailored in a cell-specific manner by changing their composition [35]. Recently, pBAEs have been used in different therapeutic applications and proven successful. Multiple applications have been described in stem cell modification or osteodifferentation, showing great promise to deliver interfering RNA (RNAi) in a safe way [36,37]. Since different backbone structures can be easily designed for pBAEs, there is a great variety of polymers to choose from [38,39]. In previous studies, results show that polymer structure has a direct influence on RNAi binding and delivery efficiency, concluding that the polymer structures C6 and C32 outperformed most of the rest [40]. These polymers show a high flexibility and can be easily tuned to fulfil specific purposes [37,41-43]. Due to this flexibility, different strategies have been tried out in order to produce nanoparticles with pBAEs. Positive results have been achieved, demonstrating that they can act as functional nanocarriers with a low toxicity [44].

Research in this area has led to an approach which focuses on creating pBAE based nanoparticles for drug delivery/gene therapy systems designed to prevent in-stent restenosis. Controlled delivery mechanisms using pBAEs are achieved in this study through the chemical bonding of nanoparticles to an initial polymer layer adhered to the stent strut. This first layer is composed of a tailored pentafluorophenyl methacrylate (PFM) coating which enables the chemical adhesion of nanoparticles, avoiding the instantaneous total release of the carriers when in contact with the medium. Nanoparticles will bond to the PFM layer, therefore, it is of great importance to produce a coating which maintains the highest number of aromatic groups as possible to enhance adhesion. While polymerization is carried out on a stent strut, the luminal part remains protected to ensure the polymer will only be deposited on the abluminal side. Chemical adhesion of the nanoparticles and their usefulness in gene therapy are also tested.

As discussed, although there have been several advancements in the understanding and treatment of atherosclerosis, there is still much more to be done. Our proposal hopes to open a pathway for pharmacological and/or gene delivery systems based on the plasma-enhanced adhesion of pBAE nanoparticles on PFM layers for stent implantation. Once a PFM layer is adhered to the substrate, pBAE nanoparticles will bond to the polymer due to its chemical nature. In this case, the carbonyl group from 
the esters of the PFM chains react with the amine group from the nanoparticles through a nucleophilic substitution reaction with pentafluorophenol acting as the leaving group [45]. This process is illustrated on Figure 1.



B
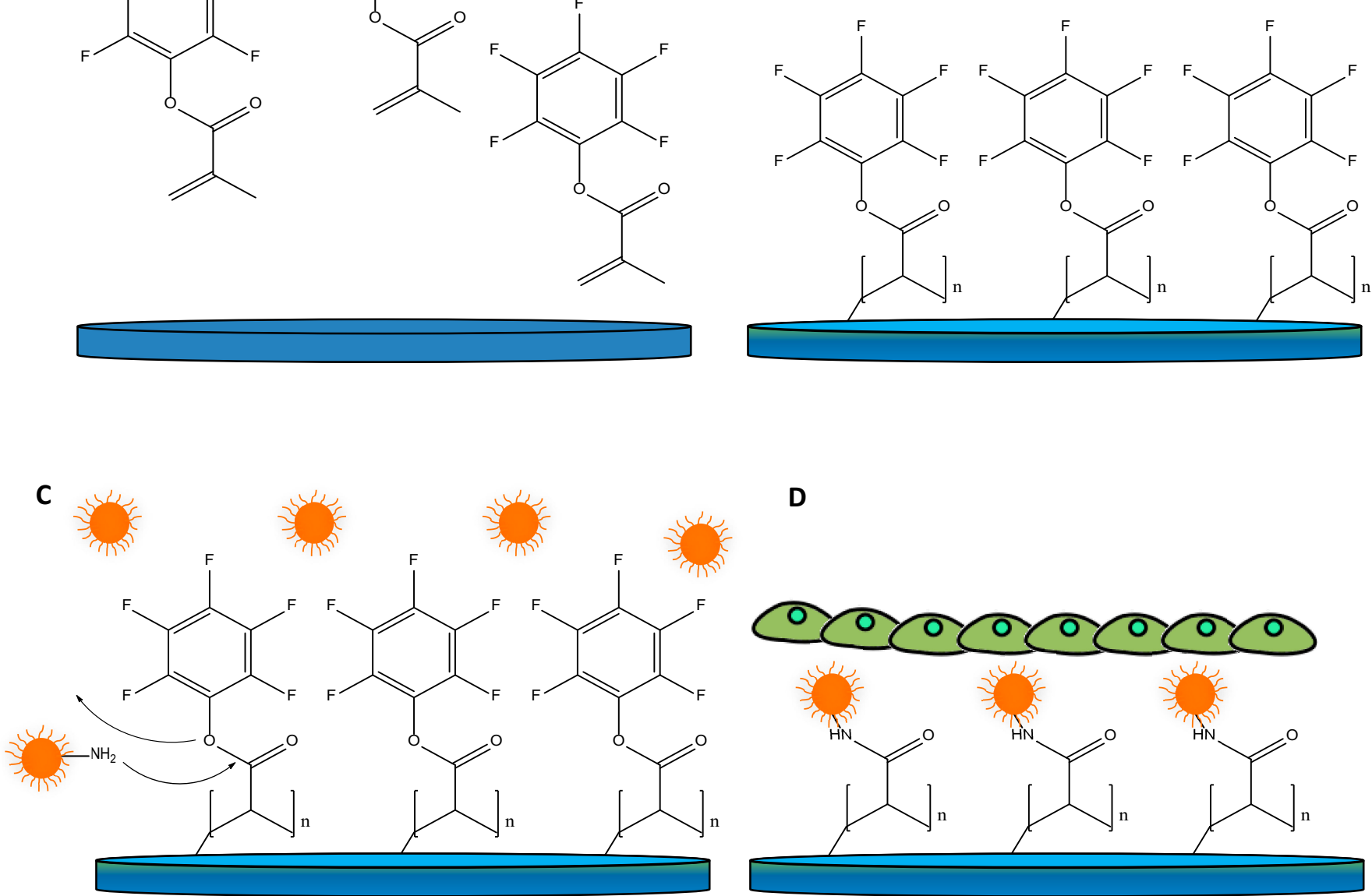

D

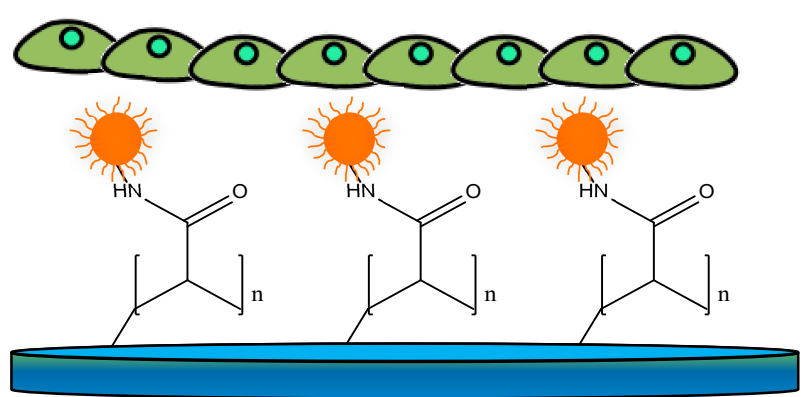

Figure 1. Schematic representation of the coating process with pentafluorophenyl methacrylate (PFM) and nanoparticles on a stent strut. Image (A) shows PFM monomer being activated in a plasma reactor. Image (B) shows PFM bonded to the sample surface after the activation process. Image (C) shows how nanoparticles bond to the PFM and image (D) shows how, after being treated with bovine serum albumin (BSA), the samples are seeded with cells.

We believe that through the bonding of carriers to a polymeric layer attached to a stent, a controlled and localized treatment can be achieved. This method will ensure the persistence of loaded nanoparticles on the stent, avoiding the carriers to be completely removed through the blood stream at first contact. When a stent is expanded at the affected area, nanoparticles will stay on the polymer matrix and mostly penetrate the target adjacent cells on to which they are pressed. This approach can 
render a higher nanocarrier efficiency due solely to the tailored release method.

\section{MATERIALS AND METHODS}

\section{Materials}

Tween-20 detergent and Bovine Serum Albumin (BSA) were obtained from Sigma-Aldrich (St. Louis, United States). Pentafluoro phenyl methacrylate (PFM) was obtained from Manchester Organics Ltd. (London, England). Dulbecco's Modified Eagle Medium was provided by Lonza (Basel, Switzerland) and fetal bovine serum by Cultek (Madrid, Spain). Glutamine and penicillin/streptomycin were obtained from Labclinics (Barcelona, Spain). Petri dishes and well plates were provided by SPL Life Sciences (Naechon-myeon, South Korea) while eppendorfs by Thermofisher (Waltham, United States). Trypsine was obtained from Gibco Life Technologies (New York, United States). Chromium Cobalt Alloy CrCo stent struts and electropolished CrCo discs $(20 \times 1 \mathrm{~mm})$ were made from alloy L605 and provided by IberHospiTex (Barcelona, Spain). Glass wafers were provided by Labbox (Barcelona, Spain). Medical silicon used to develop the silicon wafers was obtained from Nusil Technologie Europe (Mougins, France) and COS-7 cells (CRL-1651TM) were provided by ATCC (Manassas, United States).

Oligopeptide-ended pBAE were synthetized in-house through a method previously described by our research group $[35,46]$. Briefly, poly $(\beta$-amino ester)s were synthesized following a two-step procedure. First, an acrylateterminated polymer was synthesized by addition reaction of primary amines with diacrylates (at 1:1.2 molar ratio of amine:diacrylate). Then, pBAEs were obtained by end-capping modification of the resulting acrylate-terminated polymer with different kind of amine- and thiolbearing moieties. In general, oligopeptide-modified pBAEs were obtained by end-modification of acrylate-terminated polymer C32 with thiolterminated oligopeptide at 1:2.1 molar ratio in dimethyl sulfoxide. The mixture was stirred overnight at room temperature and the resulting polymer was obtained by precipitation in a mixture of diethyl ether and acetone (1:1).

For this paper, tri-arginine end-modified pBAE polymer, C32-CR3 was chosen to be used as the polymer for nanoparticle formation: A solution of C32 (0.15 g, $0.075 \mathrm{mmol})$ dissolved in dimethyl sulfoxide $(2 \mathrm{~mL})$ and a solution of Cys-Arg-Arg-Arg-CONH2.4HCl (CR3, $0.11 \mathrm{~g}, 0.15 \mathrm{mmol}$ ) in dimethyl sulfoxide $(1 \mathrm{~mL})$ were mixed and stirred overnight at room temperature. End-modified polymer CR3-C32-CR3 was obtained by precipitation in a mixture of diethyl ether:acetone (1:1).

\section{Nanoparticle Synthesis}

pBAE nanoparticles loaded with green fluorescent plasmid (GFP) were synthetized using the method we have previously described [44,46,47]. 
Briefly, pBAE stock solutions in dimethyl sulfoxide $(100 \mathrm{mg} / \mathrm{mL})$ were diluted with acetate buffer ( $25 \mathrm{mM}$ acetate buffer $\mathrm{pH}$ 5.0) at appropriate concentrations to obtain the desired Polymer-DNA ratios (w/w). An appropriately diluted pBAE (100 $\mu \mathrm{L})$ was added to a solution of green fluorescent protein vector (pGFP) $(100 \mu \mathrm{L}$ at $60 \mu \mathrm{g} / \mathrm{mL}$ in acetate buffer $25 \mathrm{mM} \mathrm{pH} \mathrm{5.0),} \mathrm{mixed} \mathrm{with} \mathrm{vortex} \mathrm{vigorously} \mathrm{for} \mathrm{a} \mathrm{few} \mathrm{seconds} \mathrm{and}$ incubated at $37^{\circ} \mathrm{C}$ for $30 \mathrm{~min}$. These nanoparticles were finally diluted in ultrapure water to achieve the desired working volume containing $1 \mu \mathrm{g} / \mathrm{well}$ of pGFP.

\section{Morphology}

Scanning electron microscopy (SEM, Jeol JSM-5310, Tokio, Japan) equipped with a microanalysis Oxford Instrument Link-Isis software (Abingdon, England) for data analysis, a profilometer (Veeco Instruments, DEKTAK 6M, New York, United States), an atomic force microscopy (AFM, Park Systems XE 100 Series, Suwon, South Korea) and an infrared (IR) spectrophotometer (Thermo Scientific, iS10, Waltham, United States) with a complementary Attenuated Total Reflection (ATR) accessory (Thermo Scientific, Smart ATR, Waltham, United States) were employed to characterize shape, thickness, rugosity and quality of the surfaces produced. To obtain SEM images, samples required a gold coating which was obtained with a mini sputter Coater (Quorum, SC7620, Lewes, UK). AFM was used with a non-contact mode. Samples were placed over a sample carrier disc and fixed with double-sided adhesive tape at room temperature in a vibration-free environment.

\section{In Vitro Transfection Assay}

COS-7 cells were cultured in Dulbecco's modified Eagle medium (DMEM) with $10 \%$ fetal bovine serum (FBS), 1\% penicillin/streptomycin and $1 \%$ glutamine. These cells were grown in Petri dishes up to $90 \%$ confluence in their culture medium at $37{ }^{\circ} \mathrm{C}$ under $5 \% \mathrm{CO}_{2}$. The cells were harvested by trypsination, collected by centrifugation ( $5 \mathrm{~min}$ at $130 \times \mathrm{g}$ ), resuspended and counted in a Neubauer chamber (Thermofisher, Waltham, United States).

After completing the synthesis of nanoparticles, which encapsulate pGFP, the glass samples with PFM were taken out of the reactor and placed in a 24 well plate. All samples were covered with a nanocarrier solution and left for two hours to ensure the chemical binding. Later, all wells were aspirated and either washed with BSA, or left unwashed. BSA was left for one hour on the selected wells before being aspirated. Cells were seeded at a density of 50,000 cells per well in 24 well plates for the glass disc samples. CrCo disc samples required seeding 100,000 cells per well in 12 well plates. Cells were left incubating in supplemented medium for 24 hours (h) before checking transfection for the first time, and later checked again at $48 \mathrm{~h}$. 
When reproducing this process with metallic stents, small variations had to be done. In this case, 6 well plates were seeded with 150,000 cells per well in $3 \mathrm{~mL}$ of supplemented medium each and left incubating for 2 days. Once accomplished, stents were coated with PFM and left for $2 \mathrm{~h}$ in a $1.5 \mathrm{~mL}$ eppendorf with the nanoparticle solution. Later, all eppendorfs were aspirated, washed with BSA and left for $1 \mathrm{~h}$ in this solution. After completing this process, stents were ready for in vitro testing. Following, stents were added to the wells to start the transfection assay. Images were taken 24 and $48 \mathrm{~h}$ after.

\section{Plasma Polymerization Reactor}

A plasma deposition apparatus which consists of a stainless-steel discharge vessel (diameter: $26 \mathrm{~cm}$, length: $24 \mathrm{~cm}$ ) parallel plate reactor was used throughout the whole experimentation. In this model the ground electrode is the reactor chamber itself, and the radiofrequency (RF) electrode is a stainless-steel plate. All the samples were treated on the central part of this plate. The RF electrode was connected to a RF pulse generator $(13.56 \mathrm{MHz})$ via a matching network. The $\mathrm{O}_{2}$ used to clean the reactor was added through a flux controller while the monomer was supplied via a standard manifold which was able to adjust gas flux using needle valves. System pressure was determined by a Pirani type vacuum meter, located between the reactor and a cold trap which was fed with dry ice. The two-stage mechanical pump (RV12 903, Edwards, GB, Bolton, England) is located after the cold trap, and evacuates the vessel to a reactor chamber at pressure of 0.1 mbar. All polymerizations were performed on silicon wafers, glass wafers, $\mathrm{CrCo}$ discs and $\mathrm{CrCo}$ stent struts. These samples were treated under pulsed plasma with a pulsed RF power ranging from 12 to $52 \mathrm{~W}$, duty cycles (DC) of 10/20, 2/12, 2/22 and 2/52 with deposition times from 5 to $65 \mathrm{~min}$. Before starting the polymerization, the monomer (PFM) and the entry lines were preheated with heating jackets up to $75{ }^{\circ} \mathrm{C}$. After preheating, monomer (PFM) flow was stabilized and fixed to achieve an internal reactor pressure of 0.35 mbar. A diagram of the reactor used can be seen in Figure 2 .

\section{RESULTS AND DISCUSSION}

Do to the unique geometry of this reactor and the variety of surfaces which were coated (glass, CrCo disks and stents), plasma polymerization conditions had to be optimized. Finding the ideal parameters for this reactor was crucial in order to ensure the maximum number of functional groups on the PFM coatings. This allowed an increase in the number of nanoparticles attached to the surface, thus accomplishing the highest therapeutic activity possible. To ensure the maximization of functional groups on PFM layers for each sample, different parameters were tested to optimize the process in our reactor, as shown in Table 1. 


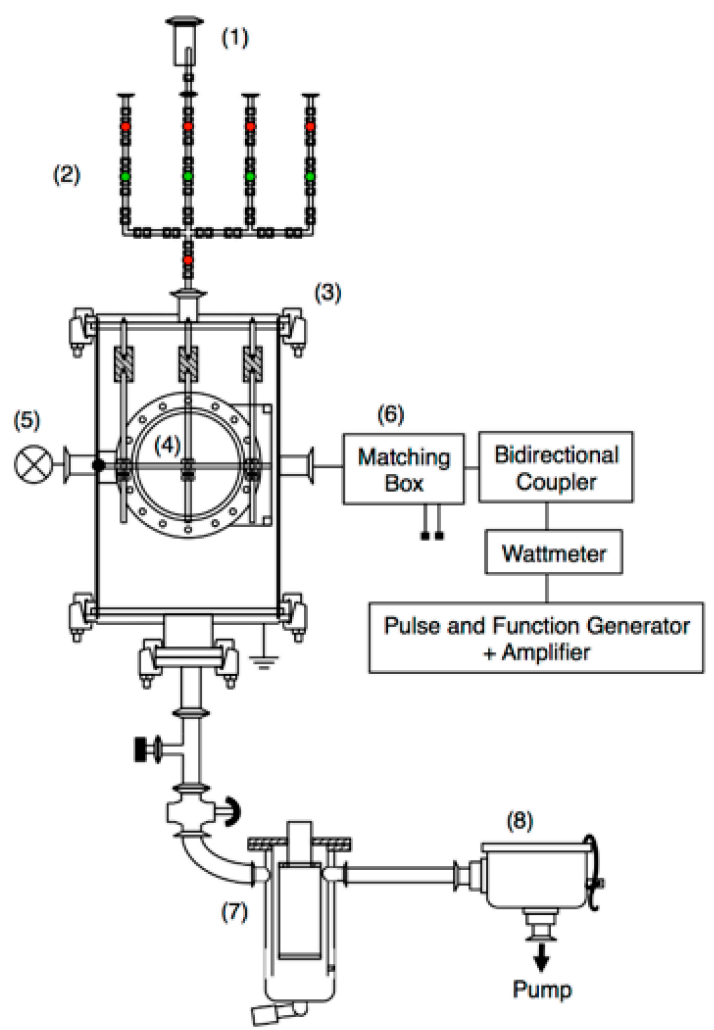

Figure 2. Schematic diagram of stainless steel vertical plasma reactor and its electrical components: (1) monomer feed, (2) gases feed, (3) cylindrical chamber, (4) holder sample, (5) pirani gauge, (6) matching box and electrical system, (7) cold trap, and (8) chemical trap.

Table 1. Three different processes are defined with a $t_{\mathrm{on}}=2 \mathrm{~ms}$ for the exception of the first one $\left(t_{\mathrm{on}}=10 \mathrm{~ms}\right)$. As this first process is the one commonly used in our laboratory it was used as a reference to compare results. In this experiment $t_{\text {process }}$ refers to the time it takes to complete a polymerization, starting when plasma is generated inside the reactor and finishing when the reactor is turned off. As expected in plasma polymerization, $\boldsymbol{C}=\frac{t_{o n}}{t_{o n}+t_{o f f}}, t_{\text {on }}$ shows the time the plasma pulse is active and $t_{\text {off }}$ indicates the time the plasma pulse is shut down. $P_{\text {peak }}$ is the input power given to the system and $P_{\text {eq }}$ is the equivalent power applied taking in account the time that the plasma pulse is active.

\begin{tabular}{cccc}
\hline Duty Cycles (DC) & $\boldsymbol{t}_{\text {process }} / \mathbf{m i n}$ & $\boldsymbol{P}_{\text {eq }} / \mathbf{W}$ & $\boldsymbol{P}_{\text {peak }} / \mathbf{W}$ \\
\hline $\mathbf{1 0 / 2 0}$ & 5 & 7.5 & 15 \\
$\mathbf{2 / 1 2}$ & 15 & 1.9 & 12 \\
$\mathbf{2} / \mathbf{2 2}$ & 30 & 1.9 & 20 \\
$\mathbf{2} / \mathbf{5 2}$ & 65 & 1.9 & 52 \\
\hline
\end{tabular}

Samples obtained must be reproducible and show a good stability of the PFM deposited on their surface. Time of reaction is also taken into account, but as a secondary objective which should be minimized. After the polymerization, each sample was stored in argon atmosphere and an infrared (IR) spectrum (Thermo Scientific, iS10, Waltham, United States) was obtained in less than $24 \mathrm{~h}$. Spectrums studied were used to obtain a ratio between the signal from the ester and the aromatic group. This ratio indicates the stability of the PFM, showing a lower value in the samples which maintain their aromatic group. Degradation of PFM results in a 
reduction of aromatic group signalling, causing the ratio (Carbonyl group/aromatic group) to increase its value. Figure 3 shows the results obtained after applying the procedure to glass and CrCo disks. On the two graphs, the same tendencies can be observed for both substrates. Plotted results were separated in three different groups labelled as A, B and C. Group A shows the highest ratio and therefore, the poorest quality PFM obtained. Although the reaction time is very short, quality of the PFM coating remains a primary target. Groups B and C obtained a very low ratio (Carbonyl group/aromatic group), although group C presented a longer reaction time. Group B shows low ratios with low and intermediate reaction times, presenting the best results out of the three groups. Among the two DC tested in group B, 2/12 is chosen as the most suitable due to its low ratio and short time of reaction.
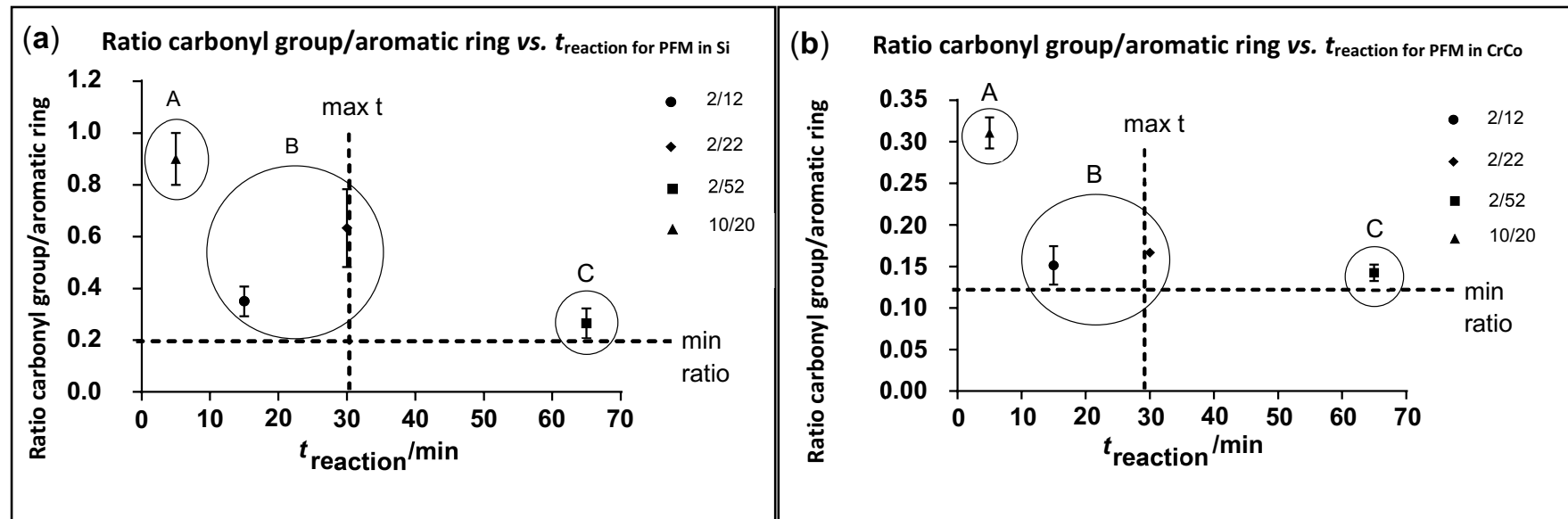

(c)



Figure 3. Graph (a) shows ratio carbonyl/Aromatic group vs. $t_{\text {reaction }}$ for Si samples. Graph (b) shows ratio carbonyl/Aromatic group vs $t_{\text {reaction }}$ for CrCo samples. These graphs show the results obtained through an optimization process elaborated to acquire an optimum coating process. A low carbonyl/Aromatic group ratio is desirable as it results in a good quality PFM coating, considering a low reaction time as a secondary target. Image (c) shows samples with PFM obtained after polymerization. First row of samples displays coated CrCo discs, while second row displays coated glass discs. Starting from left to right, the following DCs have been used: 10/20,2/12, 2/22 and 2/52. Among all DC and reaction times tested, 2/12 is selected as optimum due to the quality of the coatings produced and the low reaction time achieved.

Once a DC of 2/12 is chosen as the most appropriate, the polymerized PFM surfaces obtained on CrCo discs was characterized through SEM imaging, profilometry and AFM. Film growth rate and thickness were obtained by coating different CrCo discs at different time intervals. Profilometry was used to determine the step on these different samples. A 
film growth rate of $3.4 \mathrm{~nm} / \mathrm{min}$ and a final thickness of $79.8 \pm 4.6 \mathrm{~nm}$ were obtained after a 15 min reaction. An AFM analysis was performed on the samples rendering flat, homogeneous surfaces which show the characteristic structures of PFM coatings. Roughness average (Ra) obtained is $0.550 \mathrm{~nm}$, root mean square roughness $(\mathrm{Rq})$ is $0.644 \mathrm{~nm}$ and maximum roughness (Rmax) is $2.093 \mathrm{~nm}$, as shown in Figure 4. While Ra is the most common parameter used, Rq presents useful complementary information. Rq amplifies occasional high or low readings, while Ra simply averages them. Rmax shows the length of the highest peak obtained. Mean roughness depth (Rz) is the arithmetic mean value of the single roughness depths of consecutive sampling lengths. Elaboration of this surface morphology was achieved as a result of the experimentation with reactor geometry and the use of pulsed plasma conditions. This plasma methodology allows a controlled nucleation and film growth with a slow deposition rate, enabling the formation of films with such a low roughness.

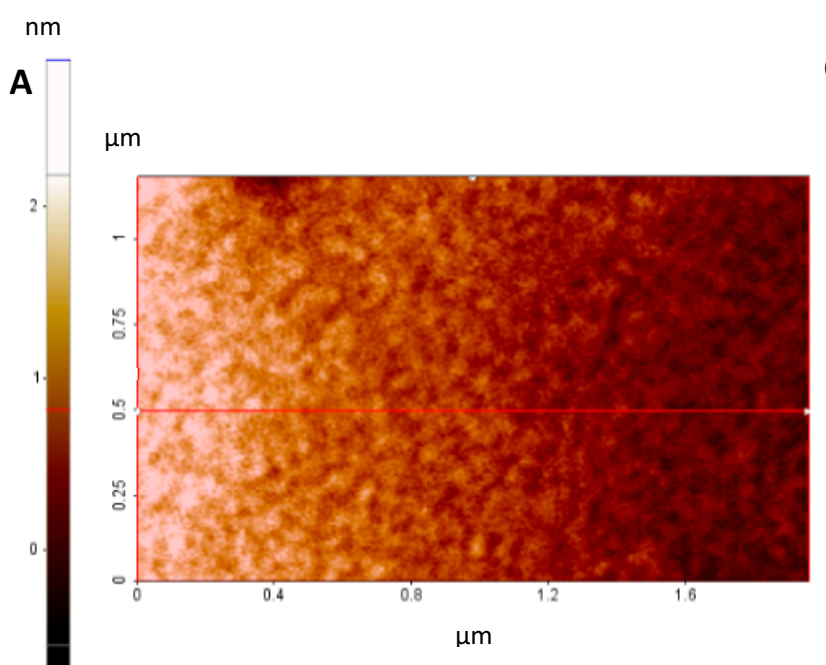

Line Profile: Red - 64

C $\mathrm{nm}$



Power Spectrum: Red - 64

$\mu \mathrm{m}$

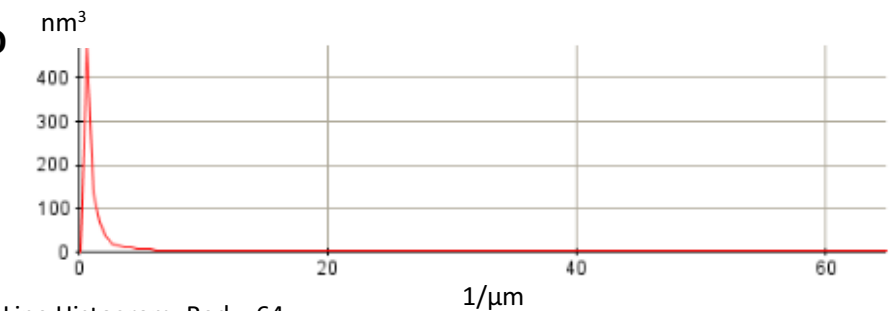

Histogram

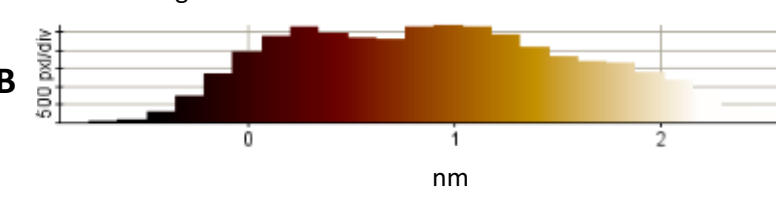

E

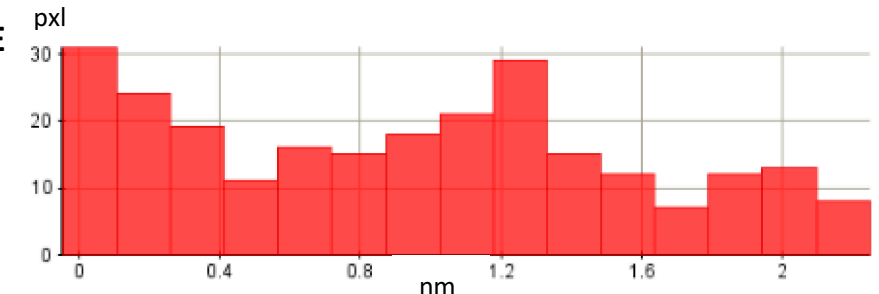

\begin{tabular}{|llllllllllll|}
\hline Line & $\operatorname{Min}(\mathrm{nm})$ & $\operatorname{Max}(\mathrm{nm})$ & $\operatorname{Mid}(\mathrm{nm})$ & $\operatorname{Mean}(\mathrm{nm})$ & $\mathrm{Rpv}(\mathrm{nm})$ & $\mathrm{Rq}(\mathrm{nm})$ & $\mathrm{Ra}(\mathrm{nm})$ & $\mathrm{Rz}(\mathrm{nm})$ & $\mathrm{Rsk}$ & $\mathrm{Rku}$ & \\
Red & -0.202 & 2.093 & 0.945 & 0.782 & 2.296 & 0.644 & 0.550 & 2.023 & -0.215 & 1.974 \\
\hline
\end{tabular}

Figure 4. AFM analysis on CrCo surfaces. (A) Characteristic worm-like structure from PFM. Additional analysis data is presented through histogram (B), line profile (C), power spectrum (D) and line histogram (E). Chart (F) summarizes the roughness data obtained. Ra and $\mathrm{Rz}$ show values much lower than $1 \mu \mathrm{m}$. This reduced roughness values are of vital importance when producing an implantable surface which has to reduce inflammatory response [48-50]. 
As shown in Figure 4, surfaces obtained show reduced values for average roughness. These results are desirable when producing a stent surface in order to avoid inflammatory response [48-50]. AFM characterization of the PFM layers showed roughness results much lower than $1 \mu \mathrm{m}(\mathrm{Ra}=0.550 \mathrm{~nm}, \mathrm{Rq}=0.644 \mathrm{~nm}$ and $\mathrm{Rz}=2.023 \mathrm{~nm})$, producing optimum quality and smooth coatings. Once the surfaces obtained had been analysed, the optimized process was ready to be used throughout the experimentation.

As a next step, a method to attach nanoparticles on to the plasma polymerized surfaces was necessary. Initial experiments were carried out on glass discs to ensure cells could grow easily on a well-known substrate. Glass waffles were coated with PFM, later taken out of the reactor, and placed in a 24 well plate. All samples were prepared as described previously in the "In Vitro Transfection Assay" section. Nanoparticles were deposited over the PFM layers and incubated for 24 and $48 \mathrm{~h}$, aiming to check for cell morphology and transfection.

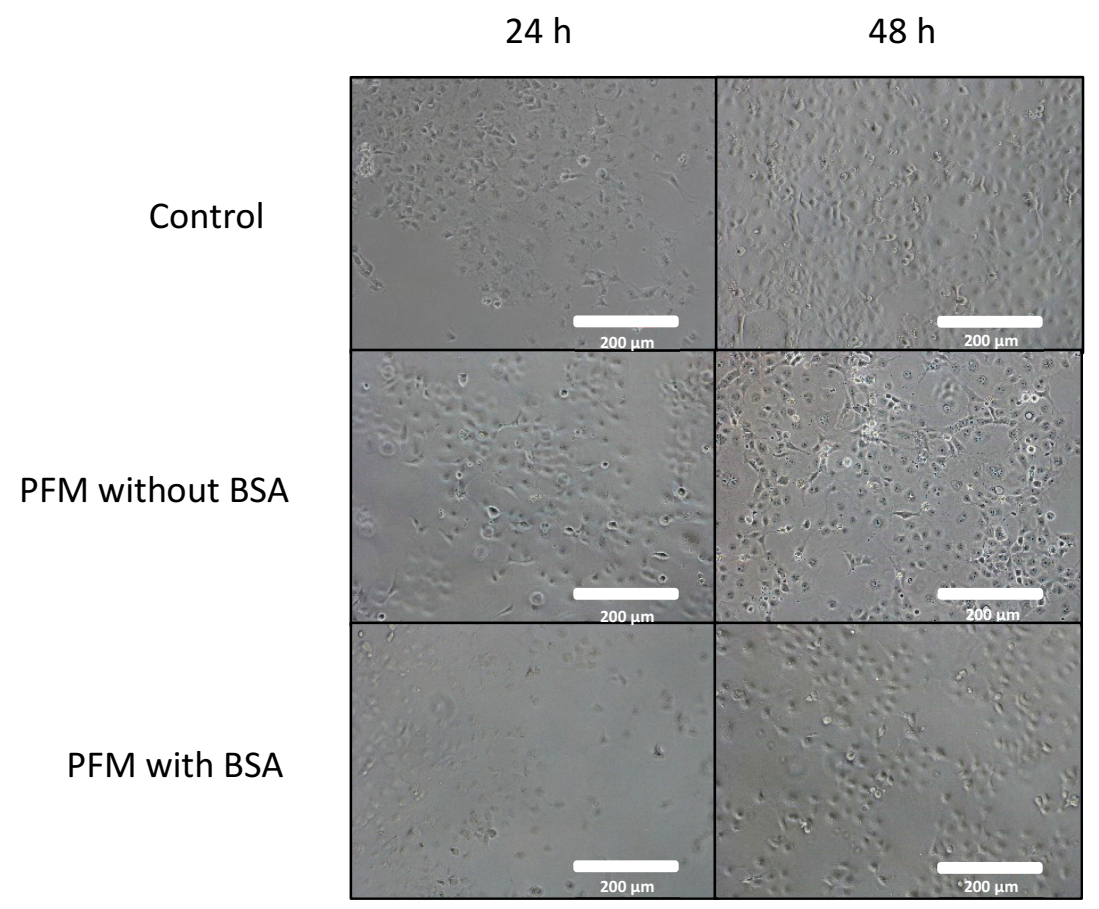

Figure 5. Bright field of various images obtained from COS-7 cells growing with PFM and with/without BSA treatment. Sites chosen for each experiment may show slight variations on initial cell count, resulting on visual diffences in cell concentrations at $48 \mathrm{~h}$. No change was appreciated in cell growth when the whole sample was inspected. Normal growth rates and typical morphologies for this cell line were observed after 24 and $48 \mathrm{~h}$ for controls and PFM with BSA samples. Slight shrinkage and blebbing of cells was observed in PFM without BSA samples. All the pictures were taken under 10× magnification. The white bar indicates $200 \mu \mathrm{m}$.

First, toxicity of the PFM layer was tested in vitro using COS-7 cells (Figure 5). In this figure, images displayed in the first row have been obtained from the control group after 24 and $48 \mathrm{~h}$. Cells have grown without any possible detrimental factor, showing an expected growth rate 
with a characteristic healthy morphology for this cell line. On the other hand, the second row shows cells growing directly on the PFM layer with signs of stressed morphologies (including shrinkage, blebbing of cells and initiation of apoptosis).

In a previous article from our group, PFM has proven to become cytotoxic six hours after its polymerization due to the elution of pentafluoro phenol [45]. Its release must be avoided, as this is a serious concern for an implantable device. For that reason, images shown in the third row of Figure 5 aim to solve this issue. This last row shows samples which have been treated to ensure there is no liberation of pentafluoro phenol from the PFM layer. Absence of pentafluoro phenol elution was achieved through the treatment of PFM layers with BSA for one hour, followed by a gentle rinse. Once this process was completed, the PFM surface was prepared to be exposed to the cells. Normal growth rates and typical morphologies for this cell line are observed after 24 and $48 \mathrm{~h}$. Results obtained conclude that this treatment allows the elimination of pentafluoro phenyl groups, found in excess on the PFM surface, avoiding cytotoxicity. This procedure renders a suitable coating for cells to grow on.

$24 \mathrm{~h}$ $48 \mathrm{~h}$

C32 nanoparticles with PFM

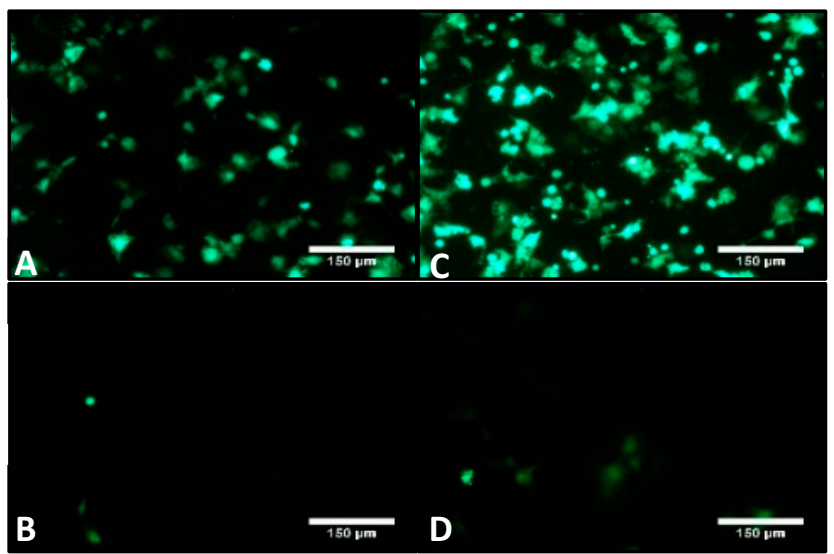

Figure 6. Images (A) and (B) are taken $24 \mathrm{~h}$ after cell seeding. Images (C) and (D) are taken at $48 \mathrm{~h}$ since cell seeding at the same area. Images (A) and (C) show transfected cells deposited over a glass disc coated with PFM and C32 nanoparticles. Samples shown on this row are coated with PFM and nanoparticles followed by a BSA treatment and a final rinse. Once this treatment is finished, cells are seeded over the samples. The results shows how, after bonding, nanoparticles stay attached to the PFM coating and are able to transfect cells 24 and $48 \mathrm{~h}$ after. Images (B) and (D) shows transfection of cells seeded on glass discs which had C32 nanoparticles deposited on top for an hour and then rinsed with BSA. Results obtained show how nanoparticles which are not bonded to PFM are easilly washed away. Controls of C32 nanoparticles with no plasmid, with and without PFM have also been performed showing black images due to absence of transfection. All the pictures were taken under 10× magnification. The white bar indicates $150 \mu \mathrm{m}$.

Once the samples were coated with PFM, glass discs were treated with C32 nanoparticles which encapsulated pGFP. Nanoparticle bonding was followed by a post treatment with BSA and a final BSA rinse. As depicted in Figure 6, samples treated with PFM, nanoparticles and BSA were able to 
transfect even after undergoing a previous rinse. This is due to the bonding process achieved, which enabled the adhesion of the nanocarriers to the surface for future release of the encapsulated pGFP. On the second row (no PFM coating applied), practically all the nanoparticles had been removed after the washing process and very low transfection was identified after 24 and $48 \mathrm{~h}$ since cell seeding. This clearly shows that without PFM, nanoparticles don't adhere to the surface and are practically completely washed away. These experiments were repeated on CrCo discs, rendering the same results.

Results obtained with disc samples led to the repetition of experimentation with cardiovascular stents. To reproduce a common stent model which could allow a selective coating, stents were masked to protect their lumen. Figure 7 presents an image of the device designed to place the stents in the reactor, while the interior area remains masked.

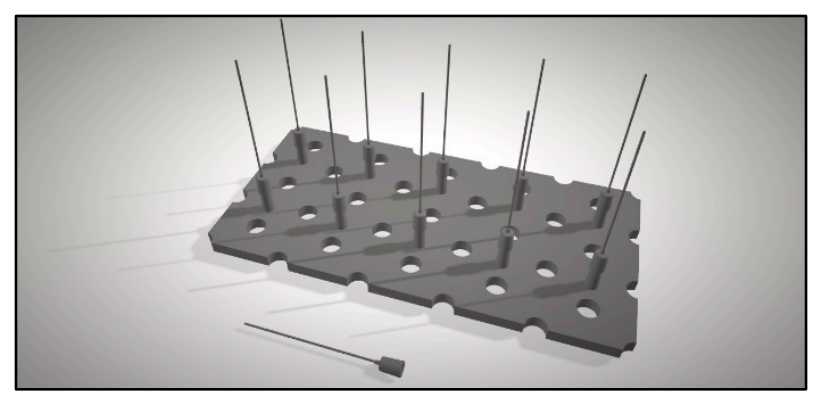

Figure 7. Image of the structure used to mount the needles which hold the stents in position inside the reactor. A small polytetrafluoroethylene plate $(100 \times 150 \times 3 \mathrm{~mm})$ was machined to obtain 10 small conic adapters which were used to place the needles. Holes were drilled into the base to match the ones in the reactor and, that way, avoid interfering with the monomer flow. Metallic needles were wrapped with polytetrafluoroethylene tape so stents could later be fitted tightly to protect their lumen.

CrCo stents were treated with PFM and then incubated with C32 nanoparticles and BSA (as described above). Part of these stents were analysed by SEM. PFM treated stents which did not undergo an immersion process with nanoparticles were used as controls. SEM pictures of both, CrCo discs and stents, are presented in Figure 8.

When CrCo disks samples were prepared, their surface was partially masked with a silicon wafer. As shown in Figure 8, when removing the wafer, a clear interface could be observed through SEM imaging. Both sides of each interface were studied through EDX analysis. Presence of polymeric coating was confirmed through the obtention of a clear carbon signal. This result was not obtained in areas which had been masked, as no carbon signal can appear over a clean CrCo surface. As shown by the SEM images, stent coatings displayed surface uniformity and smoothness throughout all the analysed samples. These results are coherent with the low average roughness of the samples obtained previously. 


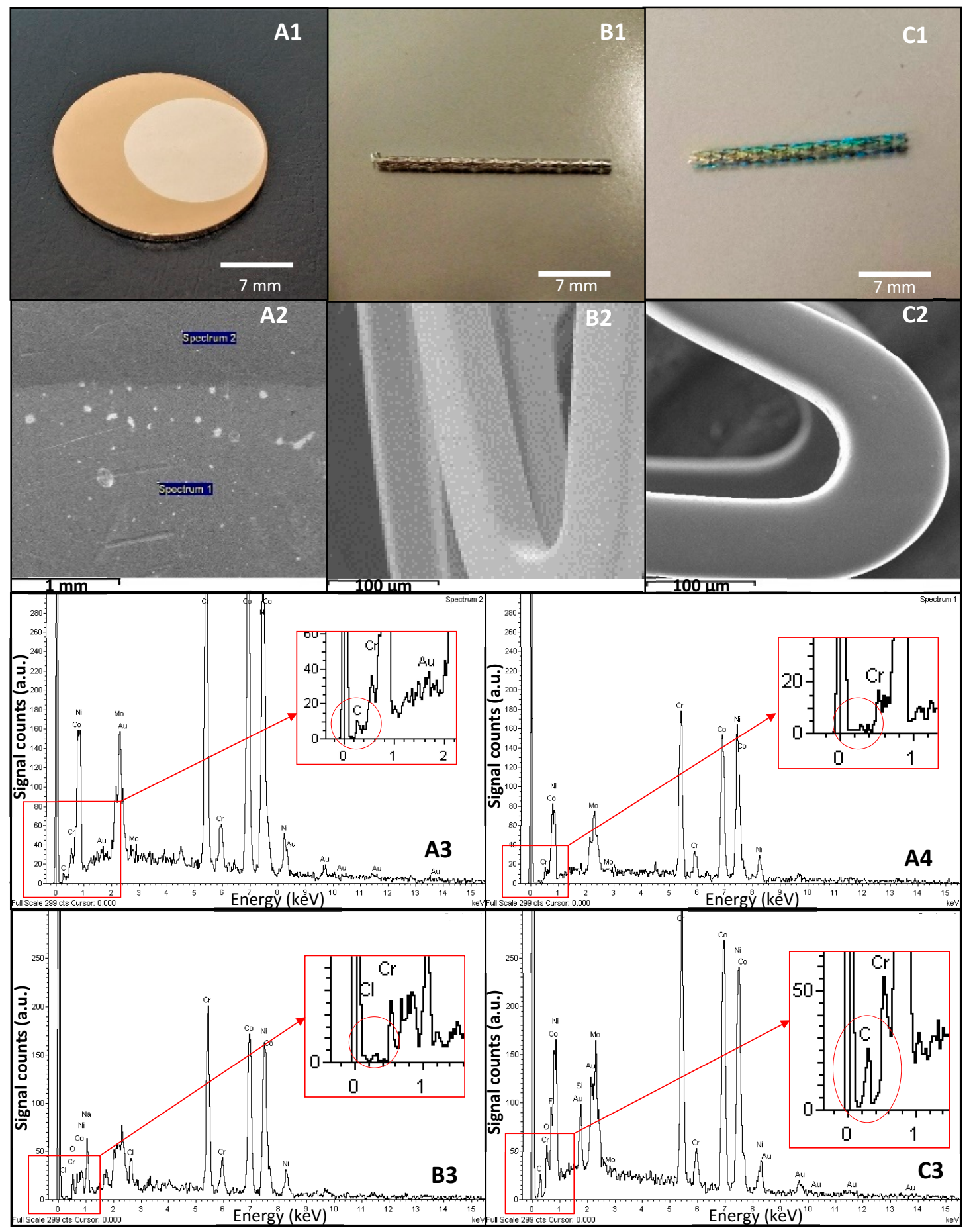

Figure 8. Images labelled as A (A1-A4) correspond to result obtained from a CrCo disc. Label B (B1-B3) corresponds to results obtained from a clean stent while label C (C1-C3) represents results acquired from a stent coated with PFM. Images A1, B1 and C1 have been taken under 1.75× magnification. Image A1 shows CrCo disc with a PFM interface. Image A2 is obtained by SEM, where a clear interface was seen. Two sites were selected for EDX analysis (spectrum 1 and spectrum 2). A3 and A4 images are the EDX spectra obtained from each site. Top right corner of A3 and A4 show their corresponding label which relates them to image A2, where analysis areas are tagged. Image $\mathbf{B} \mathbf{1}$ was obtained from a clean stent. Image $\mathbf{B} \mathbf{2}$ was obtained by SEM and image B3 is an EDX spectrum of this clean stent. C1 was taken from a stent coated with PFM. Image C2 shows an image obtained by SEM from this coated stent and image $\mathbf{C} 3$ shows its respective EDX analysis. Scale is shown at the bottom of each image (A1, A2, B1, B2, C1, and C2). Results show how the polymerization procedure rendered PFM coatings on CrCo discs and stents, which have been masked to protect specific areas. 
Following experiments required seeding COS-7 cells in 6 well plates which were left to incubate for 2 days. Once completed, cell medium was changed and stents with PFM and nanoparticles were placed on each well. This plate was left incubating for $24 \mathrm{~h}$ and images were taken in both light field and fluorescence mode.
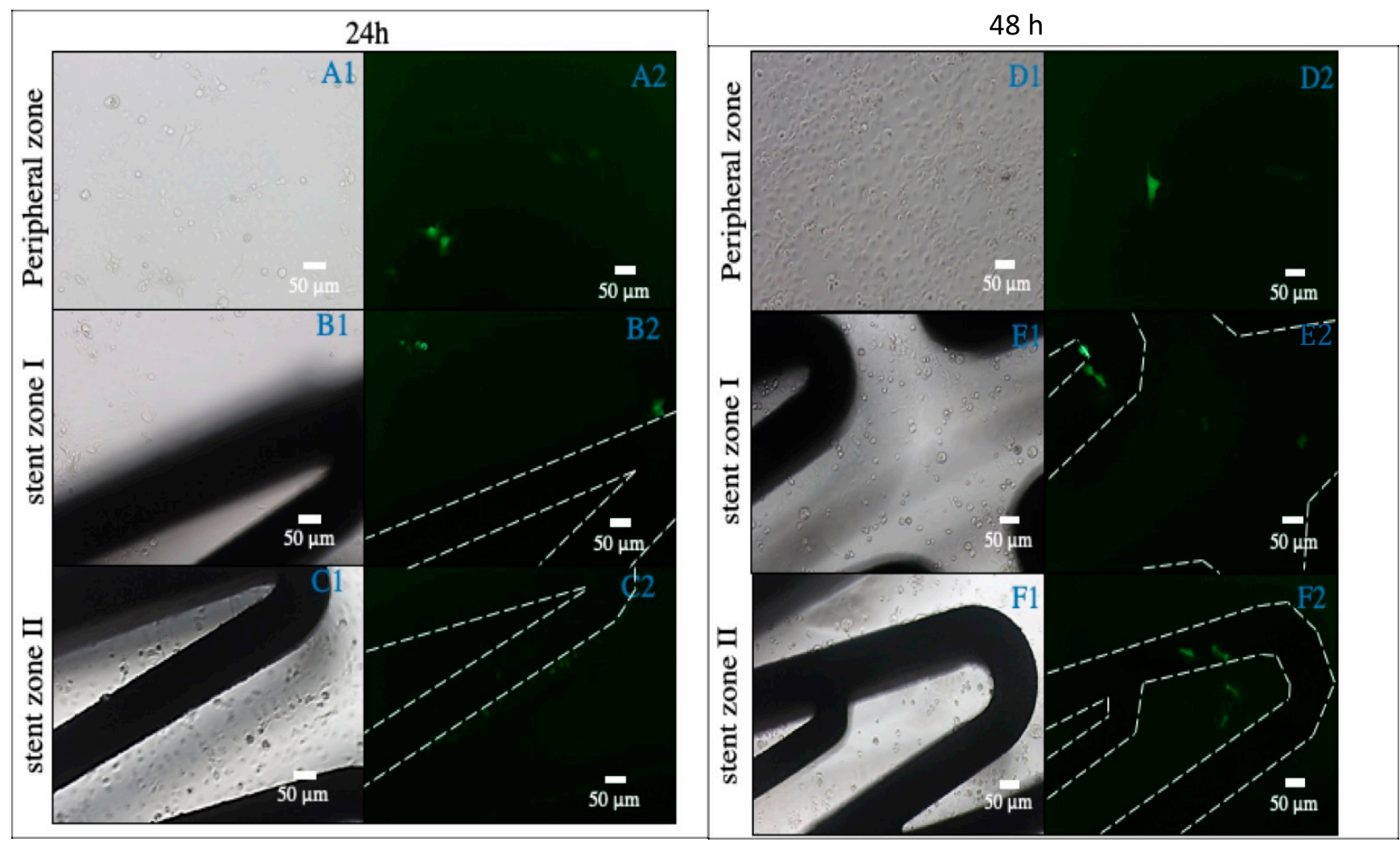

Figure 9. This figure shows results after $24 \mathrm{~h}$ and $48 \mathrm{~h}$ since cell seeding. Images A1, B1 and C1 show three different light field sites, which were obtained after leaving COS-7 cells in contact with stents, which had been coated with PFM and C32 nanoparticles for $24 \mathrm{~h}$. Images A2, B2 and C2 show respective darkfield images of the first column (A1, B1 and C1). Images D1, E1, and F1 also show three different light field sites, which were obtained in the same condition for $48 \mathrm{~h}$. Images D2, E2 and F2 show the respective darkfield images of the third column (D1, E1 and F1). Pictures where the stent strut is not seen have been taken from the surrounding area up to $5 \mathrm{~mm}$ apart from the stent (A1, A2, D1, and D2). Images B1, B2, C1, C2, E1, E2, F1 and F2 have been taken from areas where the stent structure is visible. Negative controls with stents containing nanoparticles without plasmid were not visible due to absence of transfection. Green signal shows pGFP transfection.

Microscope observation was repeated $48 \mathrm{~h}$ after and results obtained are shown in Figure 9. $24 \mathrm{~h}$ after cell seeding, some transfected cells had started growing over the stent strut as well as other cells which were not in direct contact with the stent surface. These transfected cells were identified visually due to their fluorescence. Transfection in surrounding areas is explained through a small nanoparticle release from the stents surface. As mentioned previously, nanoparticles were covalently bonded with the PFM chains during the preparation of the samples. During this process, even after bonding, nanoparticle aggregation may occur. 
Although rinsing the finished stents should eliminate most of the unreacted nanoparticles, their polymeric chains may entangle and adhere to others. When introducing one of these stents in a different medium, the liberation of aggregated nanoparticles which did not bond to PFM can transfect some adjacent cells (approximately a 10\%). This initial release was then followed by transfection of adhered nanoparticles on the stent surface. More transfection was seen $48 \mathrm{~h}$ after cell seeding, and new cells start to grow over the stents.

\section{CONCLUSIONS}

PFM has been polymerized with a high functional group retention using pulsed plasma deposition with optimum conditions in a maximum reaction time of $15 \mathrm{~min}$. Glass and CrCo surfaces have been coated with PFM, which was used to attach pBAE nanoparticles through chemical bonding. The procedure described in this article has been applied to coat glass and $\mathrm{CrCo}$ discs as well as $\mathrm{CrCo}$ cardiovascular stents. Results obtained have shown the capability of this coating to transfect COS-7 cells successfully in an initial screening. This methodology is an interesting and viable way of attaching loaded pBAE nanoparticles to stent struts for controlled release. These tailored surfaces can be used to develop pharmacological and/or gene delivery systems based on the adhesion of pBAE nanoparticles on PFM layers for stent implantation.

\section{AUTHOR CONTRIBUTIONS}

AP, VR and SB designed the experiments and interpreted the results obtained. AP performed most of the experiments described in the paper. $\mathrm{AP}, \mathrm{VR}$ and Sb wrote and corrected the paper.

\section{CONFLICTS OF INTEREST}

The authors declare that they have no conflicts of interest.

\section{FUNDINGS}

The authors would like to thank IHT company from Lliça d'Amunt (Barcelona) for its support. GEMAT group would like to thank Generalitat de Catalunya for the group support through grant SGR 20171559.

\section{REFERENCES}

1. CDC. Coronary Artery Disease (CAD). Available from: https://www.cdc.gov/ heartdisease/coronary ad.htm. Accessed 2018 Nov 1.

2. Rafieian-Kopaei M, Setorki M, Doudi M, Baradaran A, Nasri H. Atherosclerosis: process, indicators, risk factors and new hopes. Int J Prev Med. 2014;5:927-46.

3. Libby P, Libby P. History of Discovery Inflammation in Atherosclerosis. Arter Thromb Vasc Biol. 2012;32:2045-51. 
4. Yudkin JS, Kumari M, Humphries SE, Mohamed-ali V. Inflammation, obesity, stress and coronary heart disease: is interleukin-6 the link? Atherosclerosis. 2000;148:209-14.

5. Fernando A, Pérez-Vizcayno MJ, Cárdenas A, García del Blanco B, GarcíaTouchard A, López-Minguéz JR, et al. A Prospective Randomized Trial of DrugEluting Balloons Versus Everolimus-Eluting Stents in Patients with In-Stent Restenosis of Drug-Eluting Stents the RIBS IV Randomized Clinical Trial. J Am Coll Cardiol. 2015;66(1):23-3.

6. Sen H, Lam MK, Tandjung K, Löwik MM, Stoel MG, De Man FHAF, et al. Complex patients treated with zotarolimus-eluting resolute and everolimuseluting Xience V stents in the randomized twente trial: Comparison of 2-year clinical outcome. Catheter Cardiovasc Interv. 2015;85(1):74-81.

7. Kim CH, Park KWK, Kang J, Kang SH, Han JK, Yang HM, et al. TCT-753 LongTerm Comparison of Platinum Chromium Everolimus-Eluting and Cobalt Chromium Zotarolimus-Eluting Stents: Three-Year Outcome of the HOSTASSURE Randomized Trial. J Am Coll Cardiol. 2017;70(18):B318-9.

8. Iqbal J, Verheye S, Abizaid A, Ormiston J, De Vries T, Morrison L, et al. DESyne novolimus-eluting coronary stent is superior to Endeavor zotarolimus-eluting coronary stent at five-year follow-up: Final results of the multicentre EXCELLA II randomised controlled trial. EuroIntervention. 2016;12(11): e1336-42.

9. Baan J, Claessen BE, van Dijk KB, Vendrik J, van der Schaaf RJ, Meuwissen M, et al. A Randomized Comparison of Paclitaxel-Eluting Balloon Versus Everolimus-Eluting Stent for the Treatment of Any In-Stent Restenosis: The DARE Trial. JACC Cardiovasc Interv. 2018;11(3):287-97.

10. Lowik M, Van Der Heijden LC, Kok MM, Zocca P, Danse PW, Jessurun GAJ, et al. Four-year clinical outcome following randomised use of zotarolimuseluting stents versus everolimus-eluting stents in all-comers: insights from the DUTCH PEERS trial. Eur Heart J. 2018;38:620.

11. Schofer J, Schlüter M, Gershlick AH, Wijns W, Garcia E, Schampaert E, et al. Sirolimus-eluting stents for treatment of patients with long atherosclerotic lesions in small coronary arteries: Double-blind, randomised controlled trial (E-SIRIUS). Lancet. 2003;362(9390):1093-9.

12. Abou-chebl A, Bashir Q, Yadav JS. Drug-eluting stents for the treatment of intracranial atherosclerosis: initial experience and midterm angiographic follow-up. Stroke. 2005;36:e165-8.

13. Schampaert E, Cohen EA, Schlüter M, Reeves F, Traboulsi M, Title LM, et al. The Canadian study of the sirolimus-eluting stent in the treatment of patients with long de novo lesions in small native coronary arteries (C-SIRIUS). J Am Coll Cardiol. 2004;43(6):1110-5.

14. Halkin A, Stone GW. Polymer-based paclitaxel-eluting stents in percutaneous coronary intervention: A review of the TAXUS trials. J Interv Cardiol. 2004;17(5):271-82.

15. Holmes DR, Leon MB, Moses JW, Popma JJ, Cutlip D, Fitzgerald PJ, et al. Analysis of 1-Year Clinical Outcomes in the SIRIUS Trial: A Randomized Trial 
of a Sirolimus-Eluting Stent Versus a Standard Stent in Patients at High Risk for Coronary Restenosis. Circulation. 2004;109(5):634-40.

16. Ylä-Herttuala S, Baker AH. Cardiovascular Gene Therapy: Past, Present, and Future. Mol Ther. 2017;25(5):1095-106.

17. Nakano K, Egashira K, Masuda S, Funakoshi K, Zhao G, Kimura S, et al. Formulation of Nanoparticle-Eluting Stents by a Cationic Electrodeposition Coating Technology. Efficient Nano-Drug Delivery via Bioabsorbable Polymeric Nanoparticle-Eluting Stents in Porcine Coronary Arteries. JACC Cardiovasc Interv. 2009;2(4):277-83.

18. Kozuma K. Has the Development of Drug-Eluting Stents Ended With LimusEluting Stents? Circ J. 2018;82(2):330-1.

19. Verma IM, Somia N. Gene Therapy-Promises, Problems and Prospects. Nature. 1997;389(6648):239-42.

20. Somia N, Verma IM. Gene Therapy: Trials and Tribulations. Nat Rev Genet. 2000;1(November):91-9.

21. Kwon JS, Ahn YK, Jeong MH, Song SJ, Cho DL. Gene delivery stent using titanium oxide thin film coating and method for fabricating the same. United States patent US20170080131A1. 2017 Mar 23. p. 21.

22. Fernández EF, Santos-Carballal B, de Santi C, Ramsey JM, MacLoughlin R, Cryan SA, et al. Biopolymer-based nanoparticles for cystic fibrosis lung gene therapy studies. Materials. 2018;11(1):122.

23. Sanchez-Salcedo S, Vallet-Regí M, Shahin SA, Glackin CA, Zink JI. Mesoporous core-shell silica nanoparticles with anti-fouling properties for ovarian cancer therapy. Chem Eng J. 2018;340(January):114-24.

24. Baghdan E, Pinnapireddy SR, Strehlow B, Engelhardt KH, Schäfer J, Bakowsky U. Lipid Coated Chitosan-DNA Nanoparticles for Enhanced Gene Delivery. Int J Pharm. 2018;535(1-2):473-9.

25. Luderer F, Löbler M, Rohm HW, Gocke C, Kunna K, Köck K, et al. Biodegradable sirolimus-loaded poly(lactide) nanoparticles as drug delivery system for the prevention of in-stent restenosis in coronary stent application. J Biomater Appl. 2011;25(8):851-75.

26. Kumar CS. Nanomaterials: toxicity, health and environmental issues. Vol. 5. Weinheim (Germany): Wiley VCH; 2006.

27. Garg S, Serruys PW. Coronary stents: Current status. J Am Coll Cardiol. 2010;56(10 Suppl.):S1-42.

28. Abizaid A, Costa JR. New Drug-Eluting Stents an Overview on Biodegradable and Polymer-Free next-Generation Stent Systems. Circ Cardiovasc Interv. 2010;3(4):384-93.

29. Alexy RD, Levi DS. Materials and manufacturing technologies available for production of a pediatric bioabsorbable stent. Biomed Res Int. 2013;2013: 137985.

30. Nogic J, McCormick LM, Francis R, Nerlekar N, Jaworski C, West NEJ, et al. Novel Bioabsorbable Polymer and Polymer-Free Metallic Drug-Eluting Stents. J Cardiol. 2018;71(5):435-43.

31. Song L, Li J, Guan C, Jing Q, Lu S, Yang L, et al. Randomized comparison of novel biodegradable polymer and durable polymer-coated cobalt-chromium 
sirolimus-eluting stents: Three-Year Outcomes of the I-LOVE-IT 2 Trial. Catheter Cardiovasc Interv. 2018;91(November 2017):608-16.

32. Xu W, Yagoshi K, Koga Y, Sasaki M, Niidome T. Optimized polymer coating for magnesium alloy-based bioresorbable scaffolds for long-lasting drug release and corrosion resistance. Colloids Surf B Biointerfaces. 2018;163:100-6.

33. O’Connell RM, Rao DS, Baltimore D. MicroRNA regulation of inflammatory responses. Annu Rev Immunol. 2012;30:295-312.

34. Cuneo AA, Herrick D, Autieri MV. Il-19 reduces VSMC activation by regulation of mRNA regulatory factor HuR and reduction of mRNA stability. J Mol Cell Cardiol. 2010;49(4):647-54.

35. Dosta P, Segovia N, Cascante A, Ramos V, Borrós S. Surface charge tunability as a powerful strategy to control electrostatic interaction for high efficiency silencing, using tailored oligopeptide-modified poly(beta-amino ester)s (PBAEs). Acta Biomater. 2015;20:82-93.

36. Yang F, Cho S-W, Son SM, Bogatyrev SR, Singh D, Green JJ, et al. Genetic engineering of human stem cells for enhanced angiogenesis using biodegradable polymeric nanoparticles. Proc Natl Acad Sci U S A. 2010; 107(8):3317-22.

37. Tzeng SY, Hung BP, Grayson WL, Green JJ. Cystamine-terminated poly(betaamino ester)s for siRNA delivery to human mesenchymal stem cells and enhancement of osteogenic differentiation. Biomaterials. 2012;33(32):8142-51.

38. Anderson DG, Akinc A, Hossain N, Langer R. Structure/property studies of polymeric gene delivery using a library of poly( $\beta$-amino esters). Mol Ther. 2005;11(3):426-34.

39. Akinc A, Lynn DM, Anderson DG, Langer R. Parallel synthesis and biophysical characterization of a degradable polymer library for gene delivery. J Am Chem Soc. 2003;125(18):5316-23.

40. Green JJ, Langer R, Anderson DG. Yields Insight into Nonviral Gene Delivery. Acc Chem Res. 2008;41(6):749-59.

41. Jones CH, Chen M, Ravikrishnan A, Reddinger R, Zhang G, Hakansson AP, et al. Mannosylated poly(beta-amino esters) for targeted antigen presenting cell immune modulation. Biomaterials. 2015;37:333-44.

42. Tzeng SY, Wilson DR, Hansen SK, Quiñones-Hinojosa A, Green JJ. Polymeric nanoparticle-based delivery of TRAIL DNA for cancer-specific killing. Bioeng Transl Med. 2016;1(2):149-59.

43. Núñez-Toldrà R, Dosta $\mathrm{P}$, Montori S, Ramos V, Atari M, Borrós S. Improvement of osteogenesis in dental pulp pluripotent-like stem cells by oligopeptidemodified poly( $\beta$-amino ester)s. Acta Biomater. 2017;(53):152-64.

44. Borrós S, Ramos V, Segovia N, Dosta P, inventors; IQS, assignee. Modified Poly (Beta-Amino Ester)s for Drug Delivery. United States patent US 0250410 A1. 2018 Sep 6.

45. Francesch L, Borros S, Knoll W, Förch R. Surface reactivity of pulsed-plasma polymerized pentafluorophenyl methacrylate (PFM) toward amines and proteins in solution. Langmuir. 2007;23(7):3927-31. 
46. Segovia N, Dosta P, Cascante A, Ramos V, Borrós S. Oligopeptide-terminated poly( $\beta$-amino ester)s for highly efficient gene delivery and intracellular localization. Acta Biomater. 2014;10(5):2147-58.

47. Dosta P, Ramos-Perez V, Borros S. Stable and efficient generation of poly([small beta]-amino ester)s for RNAi delivery. Mol Syst Des Eng. 2018;3(4):677-89. doi: 10.1039/C8ME00006A

48. Kathuria YP. The potential of biocompatible metallic stents and preventing restenosis. Mater Sci Eng. 2006;417:40-8.

49. Mcclean DR, Eigler NL. Stent design: implications for restenosis. Rev Cardiovasc Med. 2002;3:S16-22.

50. Scott NA, Robinson KA, Nunes GL, Thomas CN, Viel K, Iii BK, et al. Comparison of the thrombogenicity of stainless steel and tantalum coronary stents. Am Heart J. 1995;129(5):866-72.

How to cite this article:

Ponce A, Ramos-Pérez V, Borrós S. Controlled Release on Cardiovascular Stents Using Plasma-Enhanced Adhesion of Biodegradable Nanoparticles. Med One. 2019;4:e190014. https://doi.org/10.20900/mo.20190014 